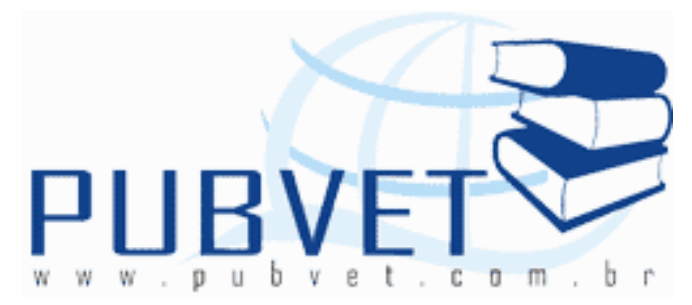

PUBVET, Publicações em Medicina Veterinária e Zootecnia.

\title{
Avaliação do uso da combinação de propofol e midazolam na contenção farmacológica e anestesia do cágado-de-barbicha Phrynops geoffroanus SCHWEIGGER, 1812 (Testudines, Chelidae)
}

André Luiz Quagliatto Santos ${ }^{1}$, Sérgio Rodrigo Pereira de Oliveira ${ }^{2}$, Árthur Paulino Sanzo Kaminishi ${ }^{2}$, Mariana Batista Andrade ${ }^{3}$, Lorena Tannús Menezes ${ }^{2}$, Rogério Rodrigues de Souza ${ }^{2}$, Caio Henrique Ferreira ${ }^{2}$, Liliane Rangel Nascimento ${ }^{2}$, Flávio Machado de Moraes $^{1}$

Laboratório de Ensino e Pesquisa em Animais Silvestres - LAPAS, FAMEV/UFU, e-mail: quagliatto@famev.ufu.br 1. Docente. 2. Mestrandos. 3. Doutoranda.

\section{Resumo}

O objetivo desta pesquisa foi determinar um protocolo eficaz e seguro para

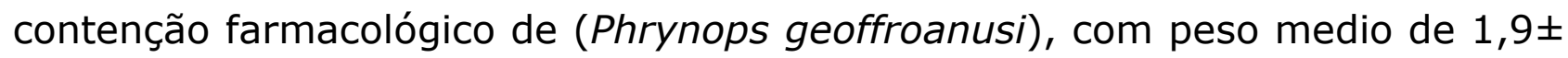
$0,77 \mathrm{Kg}$, clinicamente saudáveis, sendo 02 machos e 18 femeas, capturando no rio uberabinha no município de Uberlândia - MG sob licença M 032/2006 IBAMA-RAN, foram alojados em tanque com água corrente e aquecedores com temperatura da agua em media de $27^{\circ} \mathrm{C}$. Os quelônios foram divididos em dois grupos de 10 animais: $\mathrm{G} 1$ - midazolam $(2,0 \mathrm{mg} / \mathrm{Kg} \mathrm{IM})$ e propofol $(5,0$ $\mathrm{mg} / \mathrm{Kg} \mathrm{IV}$ ) e $\mathrm{G} 2$ - midazolam (2,0 mg/Kg IM) e propofol $2,5 \mathrm{mg} / \mathrm{Kg} \mathrm{IV})$. 0 midazolam foi aplicado no membro torácico esquerdo e o propofol foi injetado por via direta no seio vertebral cervical. Observou-se os parâmetros anestésicos: locomoção, relaxamento muscular, resposta aos estímulos nos 
membros torácicos e na cauda, facilidade de manipulação e batimentos cardíacos, nos tempos 0 e 5, 10, 20, 30, 45, 60, 90, 120, 150 e 180 minutos após as injeções. Não houve estatística significativa entre dois grupos, portanto este protocolo não é indicado para procedimentos cirúrgicos em Phrynops geoffroanus.

Palvras-chave: Anestesia, midazolan, propofol

\title{
Evaluation of the use of the combination of propofol and midazolam in the chemical restraint and anesthesia of the turtle-de-beard Phrynops geoffroanus Schweigger, 1812 (Testudines, Chelidae)
}

\begin{abstract}
The objective of this research was to determine a protocol for effective and safe pharmacological restraint (Phrynops geoffroanusi), with average weight of $1.9 \pm 0.77 \mathrm{~kg}$, clinically healthy, 02 males and 18 females, capturing in Uberabinha river in the city of Uberlândia - MG under license $M{ }^{\circ}$ 032/2006 IBAMA-RAN, were housed in a tank with running water and water heaters with an average temperature of $27^{\circ} \mathrm{C}$. the turtles were divided into two groups of 10 animals: G1 - midazolam (2.0 mg / kg IM) and propofol (5.0 mg / kg IV) and G2 - midazolam (2.0 mg / kg IM) and propofol $2.5 \mathrm{mg} / \mathrm{kg}$ IV). Midazolam was administered in the left forelimb and propofol was injected directly in the cervical vertebral sinus. There was the anesthetic parameters of movement, muscular relaxation, in response to stimuli forelimbs and tail, ease of handling and heart rate, at 0 and 5, 10, 20, 30, 45, 60, 90, 120, 150 and 180 minutes after the injections. There was no statistically significant between two groups, so this protocol is not indicated for surgical procedures in Phrynops geoffroanus.
\end{abstract}

Keywords: anesthesia, midazolam, propofol

\section{INTRODUÇÃO}


Os animais silvestres foram alvo de exploração desde os primórdios dos tempos, servindo como fonte protéica na alimentação humana. A civilização sobrevive, em certas regiões do pais, graças á exploração da natureza, cujos estoques, não respotos, são vulneráveis ao esgotamento, além disso, a fauna nativa brasileira desperta grande interesse econômico nas populações ribeirinhas que, devido á precária condição de vida aliada á baixa renda familiar e nível cultural educacional, têm direcionado a caça de subsistência para o comercio ilegal (IBAMA, 2004).

Aproximadamente $20 \%$ das 278 espécies de quelônios do mundo ocorrem na América do Sul, representando oito famílias (Dermochelydae, Cheloniidae, Chelydridae, Emydidae, Kinosternidae, Testudinidae, Podocnemidae e Chelidae). Dessas famílias Chelidae, cujos representantes típicos são os animais conhecidos popularmente como cágados, é a mais rica, contando com 23 espécies, das quais 19 ocorrem no Brasil (SOUZA, 2004).

Segundo Orr (1986), a classe dos répteis vem sofrendo grandes perdas de espécies no decorrer da sua historia, pois das 16 ordens existentes no passado, restaram apenas três: Testudines, Crocodilia e Squamata, tendo sido as outras extintas.

A ordem Testudines é constituída por tartarugas, cágados e jabutis. Esses animais possuem diferentes habitats: espécies exclusivamente terrestres como os jabutis (cx: Geochelonia SP); espécie exclusivamente terrestres como os jabutis (cx: Phrynops SP); espécies exclusivamente marinhas como as tartarugas (ex Caretta caretta) e ainda espécie que vivem em ambientes terrestre e de água doce, como a aperema (Rhynaclemmys punctularia) (GARCIA-NAVARRO;PACHALY, 1994).

Os quelônios apresentam o corpo inteiramente coberto por um casco, ou seja, carapaça na porção dorsal e o plastrão na porção ventral do corpo, que são fortes, de onde somente a cabeça, os membros e cauda emergem. Os dentes foram perdidos na escala evolutiva e as mandíbulas são cobertas por placas córneas e aguçadas (VILLE, WALKER JúNIOR; BARNES, 1985). 
Pough et al. (1999) informam que os cágados possuem carapaças baixas que oferecem pequena resistência aos deslocamentos na água, e membros anteriores modificados em remos auxiliando sua locomoção. Segundo Ernest e Barbour (1989), o cagado-de-barbicha (Phrynops geoffroanus), possui uma carapaça achatada e larga com coloração preta ou cinza esverdeada. O plastrão possui um entalhe anal e tem coloração vermelha rosada com manchas pretas irregulares. A cabeça é larga e lisa, com preta, linhas brancas e irregulares. A parte ventral é branca com linhas e pontos pretos. A característica mais proeminente dessa espécie é uma linha preta e larga que se estende longitudinalmente pelo olho, também possui um par de "barbelas" no queixo com a extremidade preta, diferente da Phrynops hilarri onde as extremidades são brancas. Os membros são cinza com manchas brancas. É uma espécie pequena, encontrada desde a Colômbia, Venezuela, Guiana, Extremo Sul do Paraguai até sudeste, centro-oeste e nordeste do Brasil. Essa espécie é freqüentemente nos rios, lagos com correnteza lenta.

Para Orr (1986), a anestesia de animais selvagens torna-se cada vez mais uma rotina na medicina veterinária, devido á maior procura por estes animais como companhia. Dentre os repteis, que por suas particularidades a anátomo - fisiológicas, constituem-se uma fonte de estudos para vários campos do conhecimento como, por exemplo, a anestesiologia.

Pough et al. (1999), a anestesia de um réptil bem sucedida requer paciência e planejamento. Parâmetros como dor são difíceis de serem avaliados na maioria deles. Estes animais são capazes de resistir á perturbações fisiológicas que matariam facilmente um mamífero ou uma ave.

O objetivo desta pesquisa foi determinar um protocolo eficaz e seguro para a contenção farmacológica e anestesia de Phrynops geoffroanus, utilizando a associação midazolam e propofol.

\section{Material e Método}


O Trabalho experimental foi realizado no laboratório de Pesquisa em Animais Silvestres da Faculdade de Medicina Veterinária da Universidade Federal de Uberlândia, no mês de agosto de 2006, onde as condições climáticas eram de tempo seco, sendo que a temperatura foi aferida pelo termômetro de máxima e mínima, com média de $27^{\circ} \mathrm{C}$.

\section{Animais Experimentais}

Os 20 cagados foram capturados no rio Uberabinha $\left(18^{\circ} 55^{\prime} 42,1^{\prime \prime} \mathrm{S}\right.$ e $48^{\circ}$ 17 '35,4" W) no município de Uberlândia - MG, sob licença N 032/2006 IBAMA-RAN e alojados em tanque com água corrente e aquecedores, mantendo a temperatura da água em media de $27^{\circ} \mathrm{C}$. Posteriormente, os animais foram pesados na balança pesadora simples ${ }^{1}$, com peso médio de 1,9 $\pm 0,77 \mathrm{Kg}$, clinicamente saudáveis, sendo 02 machos e 18 fêmeas. Todos os dados referentes ao individuo estudado foram anotados em formulário individual (Anexos 1 e 2 ), que correspondia à numeração colocada na superfície dorsal da carapaça.

\section{Grupos experimentais}

Utilizam-se 20 cágados da espécie Phrynops geoffroanus, divididos em 2 grupos, Cada grupo recebeu a seguinte associação anestésica:

- Grupo 1: midazolam² 2mg/Kg/IMe propofol ${ }^{3} 5 \mathrm{mg} / \mathrm{Kg} / \mathrm{IV}$;

- Grupo 2: midazolam 2mg/Kg/IM e propofol 2,5/Kg/IV.

O midazolam foi injetado no membro torácico e o propofol foi injetado por via direta no seio vertebral cervical (Fotografia 10), mediante prévia anti-sepsia e uso de seringas estéreis descartáveis ${ }^{4}$ de $3 \mathrm{ml}$ agulhas descartáveis ${ }^{5}$, para aplicação das drogas.

\footnotetext{
${ }^{1}$ Balança para cozinha, Yara, Guaianazes, SP.

${ }^{2}$ Dormire, Cristália, Farma, Itapira, SP.

${ }^{3}$ Provive 1\%, Core Healthcare Limited, Índia.

${ }^{4}$ Embramac, Itajaí, SC.

${ }^{5}$ Embramac 0,70 x 25 21G1, Itajaí, SC.
} 
Após a aplicação da droga, os animais foram observados quanto aos parâmetros (Locomoção, relaxamento muscular, estímulos dolorosos no membro torácico direito e cauda) e seus graus de sedação (1,2 ou 3) (Fotografia 6)

Os parâmetros foram observados nos determinados tempos: 5, 0, 15 minutos para o acompanhamento da aplicação da medicação pré-anestésica (MPA) (Anexo 1) e os tempos 0, 5, 10, 20, 30, 45, 60, 120, 150, 180 minutos para o acompanhamento após a aplicação do propofol, sendo que tempo zero foi considerado o momento da administração das drogas. Os parâmetros avaliados foram os seguintes:

1) Locomoção: capacidade de se locomover normalmente, dificuldade de locomoção e ausência de locomoção;

2) Relaxamento muscular: capacidade de manter a cabeça elevada, facilidade de estender os membros e de abrir a boca do animal

3) Manipulação: facilidade de manipular manualmente (flexão e extensão) a cabeça, os membros e a cauda. Facilidade de abrir a boca do animal (Fotografia 5)

4) Estimulo do membro torácico direito: pinçamento das falanges com pinça hemostática Kelly curva de $16 \mathrm{~cm}$ (dor profunda), ambos na segunda trava da pinça (Fotagrafia 2)

5) Estímulo da cauda: pinçamento cutâneo (fotografia 3)

6) Freqüência cardíaca: aferição dos batimentos cardíacos pelo Doppler vascular $^{6}$ nos tempos $0,10,30,60,120$ e 180 minutos;

7) Reflexos protetores oculopalpebrais: teste da presença de reflexos protetores oculopalpebrais por toque na córnea.

Um escore subjetivo de "um" para efeito mínimo. E "três" para profundo efeito foi utilizado para três primeiros parâmetros. Para os testes de sensibilidade dolorosa, a resposta ao pinçamento foi considerada escore "ZERO" e á

\footnotetext{
${ }^{6}$ Modelo DV-20, Microem, Ribeirão Preto, SP.
} 
ausência da resposta ao pinçamento foi considerado escore "um". Para o primeiro parâmetro, locomoção, o escore "Três" foi atribuído aos animais que permaneciam totalmente imóveis, "dois' para os animais que conseguiam se arrastar e "um" para os animais que se locomoviam normalmente. Para o segundo parâmetro, relaxamento muscular, o escore "Três" foi atribuído aos animais que se mantinham com os membros e cabeça distendidos, sem apresentar moimentos de defesa (fuga) na presença do pesquisador e que não tinham capacidade de manter sua cabeça elevada ao solo. Em contrapartida escore "um" foi atribuído aos animais quando apresentavam retração de cabeça e membros á manipulação.

A facilidade de manipulação foi avaliada pela capacidade de manipular manualmente (flexão e extensão) a cabeça, os membros e a cauda. Além da facilidade de abrir a boca do animal. Escore "Três" para maior facilidade e "um" para menor.

Os parâmetros estímulo do membro torácico direito, estímulo da cauda e reflexos protetores oculopalpebrais foram avaliados como 0 presença e 1 ausência de reflexos. Os repteis foram considerados anestesiados quando 0 somatório de seu escore era 11 ; e considerados recuperados quando suas atividades se aproximavam ás de antes da administração das drogas. O tempo entre a administração dos anestésicos e a recuperação dos animais foi observado.

IV. Monitoramento da temperatura ambiental e umidade.

A temperatura ambiental e umidade foram monitorados com auxilio do termo-higrômetro ${ }^{7}$ que estava disposto no mesmo plano dos animais experimentais. Esse monitoramento foi realizado durante todo o período da anestesia.

\footnotetext{
${ }^{7}$ Incoterm, Porto Alegre, RS.
} 
IV. Monitoramento da freqüência cardíaca

Os batimentos cardíacas das Phrynops geoffroanus foram monitorados com o aparelho Doppler Vascular Eletrônico nos tempos $0^{\prime}, 10^{\prime}, 30^{\prime}, 60^{\prime}, 120^{\prime}$ e 180 (Fotografia 7)

VI. Analise estatística dos dados

Com o objetivo de verificar a existência de diferenças estatísticas entre os resultados obtidos com midazolam $2 \mathrm{mg} / \mathrm{Kg}$ IM e propofol 2,5mg/Kh IV e midazolam $2 \mathrm{mg} / \mathrm{kg}$ IM propofol $5 \mathrm{mg} / \mathrm{kg}$ IV, foi aplicado o teste $U$ de MannWhitney (SIEGEL, 1975), aos dados encontrados nos diversos tempos em que foram efetuadas os experimentos.

O nível de significância foi estabelecido em 0,05 em uma prova bilateral.

VII. Confecção das fotografias

Foram confeccionadas fotografias para ilustração da aplicação do anestésico (fotografia 1), da avaliação do estimulo no membro direito (fotografia 2), da avaliação do estímulo da cauda (Fotografia 3), da avaliação do relaxamento muscular (Fotografia 4), da avaliação da Facilidade de manipulação (Fotografia 5), da sedação (fotografia 6) e da freqüência cardíaca (fotografia 7).

\section{Resultados}

Durante o experimento, foi registrada que a temperatura média ambiental esteve entre os 28 e $31^{\circ} \mathrm{C}$ e a umidade apresentou valor médio de $22 \%$. 
Dos 20 repteis estudados, apenas 17 obtiveram escore 3 (efeito desejado) para os parâmetros locomoção, relaxamento muscular e facilidade de manipulação em até 05 minutos após a administração dos anestésicos. No G2, 04 animais em anestesia. No G1, 05 obtiveram escore máximo. Todos os animais foram acompanhados por 48 horas após a administração dos anestésicos.

A estatística foi feita de acordo com a média de tempo (minutos) em que os animais mantiveram-se no maior escore para cada parâmetro. Os animais que não obtiveram escore 11 também foram analisados dentro do seu grupo.

Pode-se observar que o G1 obteve maior média em minutos para os parâmetros: tempo total de retorno ás condições pré-anestesicas (289 min.), facilidade de manipulação (75,5 min,) e tempo de anestesia, ou seja, escore 11 (25,5 min.) (tabela 01).

Tabela 01: Médias de tempo, em minutos, de cada grupo, para os parâmetros avaliados após a aplicação do propofol.

\begin{tabular}{|l|c|c|}
\hline \multicolumn{1}{|c|}{ PARÂMETRO } & \multicolumn{2}{c|}{ GRUPOS } \\
\hline & G1 & G2 \\
\hline Retorno & 289 & 275 \\
\hline Sedação & 101 & 101,5 \\
\hline Locomoção & 101 & 101,5 \\
\hline Relaxamento Muscular & 88 & 87 \\
\hline Facilidade de Manipulação & 75,5 & 59,5 \\
\hline Escore II & 25,5 & 15 \\
\hline
\end{tabular}

Com o uso de midazolam, foi observado o bom relaxamento muscular e facilidade de manipulação em ambos os grupo (Tabela 01).

Com relação ao estimulo no membro torácico, em G1 e G2, 06 e 07 animais não apresentaram respostas ao pinçamento, respectivamente. Já em 
relação ao estimulo da cauda, em G1 e G2, 04 e 02 animais não apresentaram resposta, respectivamente.

Os Grupos G1 e G2 não apresentavam diferença significativa para os parâmetros relaxamento muscular, estimulo do membro direito, estimulo da cauda, manipulação e reflexos protetores oculopalpebrais, nos diferentes tempos ( $p>0,05)$, conforme demonstrado nos gráficos 2,3,4,5 e 7. Entretanto, para os parâmetros locomoção e freqüência cardíaca, observou-se diferença $(p<0,05)$ no tempo 10 para propofol $2,5 \mathrm{mg} / \mathrm{Kg}$ IV e no tempo 0 para propofol $5 \mathrm{mg} / \mathrm{Kg} \mathrm{IV}$, respectivamente, conforme demonstrado nos gráficos 1 e 6 .



Gráfico 1: Médias dos escores de perda da capacidade de locomoção nos diferentes tempos, em Phynops geoffroamus anestesiadas com as associações midazolam $2 \mathrm{mg} / \mathrm{kg}$ IM e propofol $5 \mathrm{mg} / \mathrm{kg}$ IV e midazolam 2 $\mathrm{mg} / \mathrm{kg}$ IM e propofol $2,5 \mathrm{mg} / \mathrm{kg} \mathrm{IV}$. 
SANTOS, A.L.Q. et al. Avaliação do uso da combinação de propofol e midazolam na contenção farmacológica e anestesia do cágado-de-barbicha Phrynops geoffroanus SCHWEIGGER, 1812 (Testudines, Chelidae). PUBVET, Londrina, V. 6, N. 12, Ed. 199, Art. 1337, 2012.

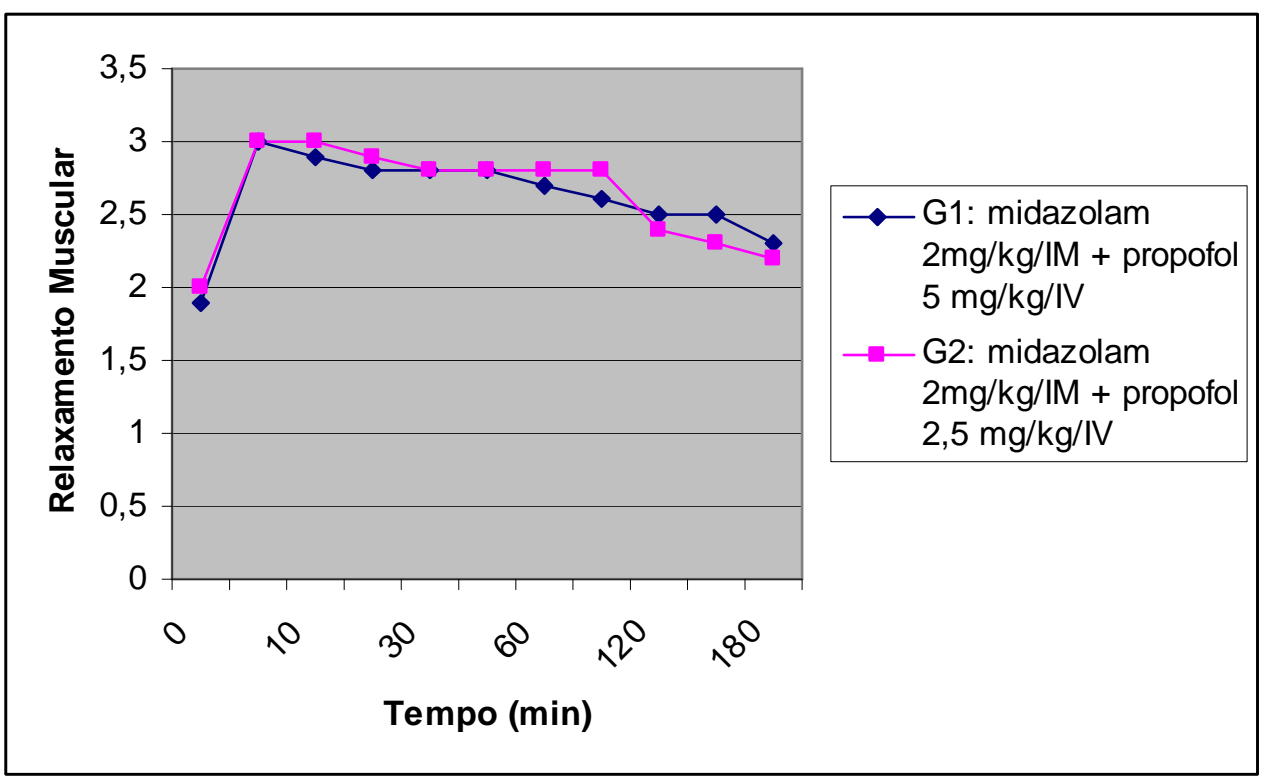

Gráfico 2: Médias dos escores de relaxamento muscular nos diferentes tempos, em Phynops geoffroamus anestesiadas com as associações midazolam $2 \mathrm{mg} / \mathrm{kg}$ IM e propofol $5 \mathrm{mg} / \mathrm{kg}$ IV e midazolam $2 \mathrm{mg} / \mathrm{kg} \mathrm{IM}$ e propofol $2,5 \mathrm{mg} / \mathrm{kg} \mathrm{IV}$.

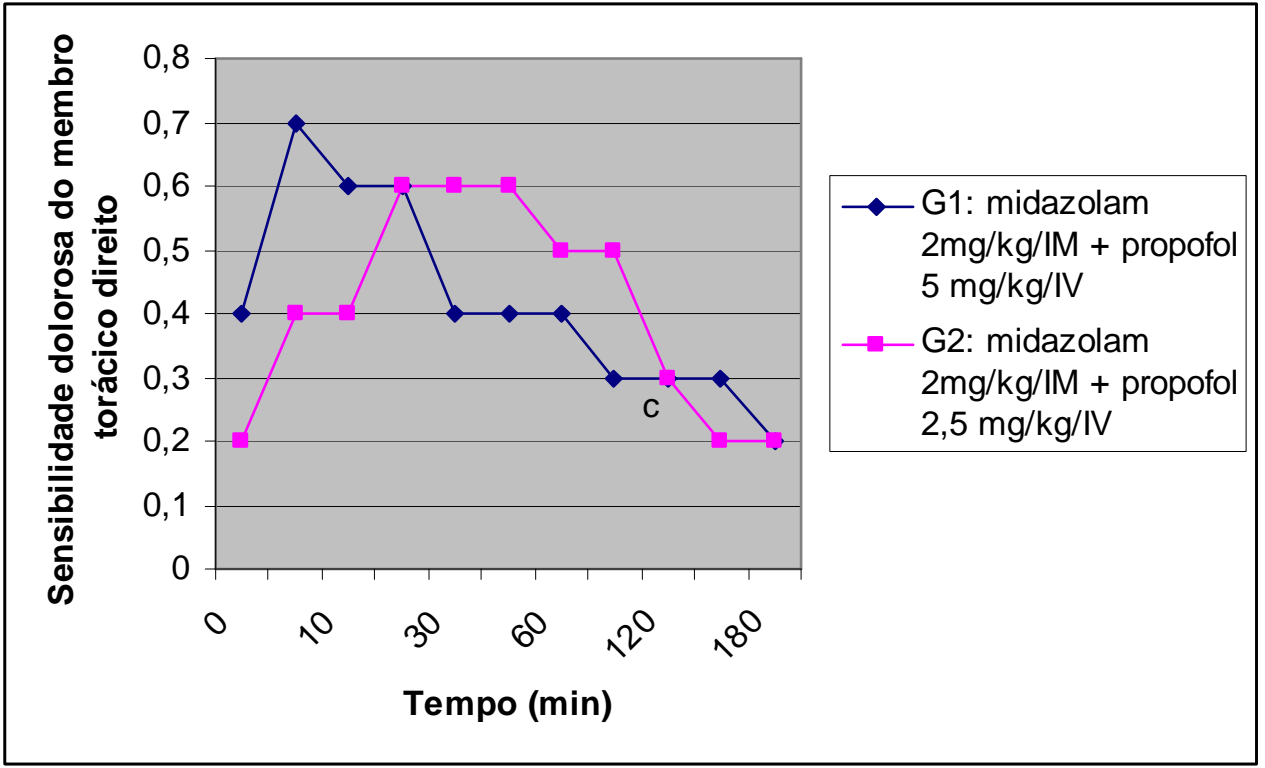

Gráfico 3: Médias dos escores de estímulos no membro torácico direito nos diferentes tempos, em Phynops geoffroamus anestesiadas com as associações midazolam $2 \mathrm{mg} / \mathrm{kg} \mathrm{IM}$ e propofol $5 \mathrm{mg} / \mathrm{kg}$ IV e midazolam 2 $\mathrm{mg} / \mathrm{kg}$ IM e propofol $2,5 \mathrm{mg} / \mathrm{kg}$ IV. 
SANTOS, A.L.Q. et al. Avaliação do uso da combinação de propofol e midazolam na contenção farmacológica e anestesia do cágado-de-barbicha Phrynops geoffroanus SCHWEIGGER, 1812 (Testudines, Chelidae). PUBVET, Londrina, V. 6, N. 12, Ed. 199, Art. 1337, 2012.

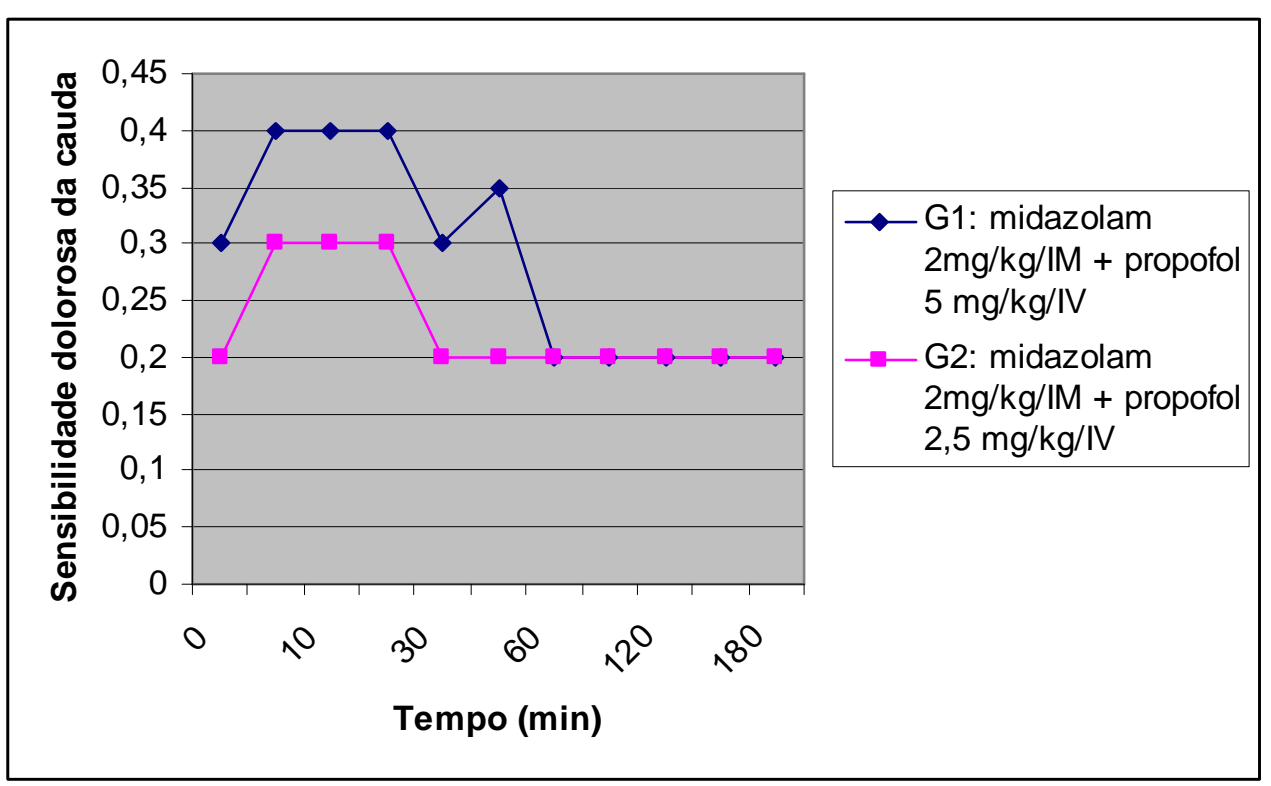

Gráfico 4: Médias dos escores do estimulo da calda nos diferentes tempos, em Phynops geoffroamus anestesiadas com as associações midazolam $2 \mathrm{mg} / \mathrm{kg} \mathrm{IM}$ e propofol $5 \mathrm{mg} / \mathrm{kg}$ IV e midazolam $2 \mathrm{mg} / \mathrm{kg} \mathrm{IM}$ e propofol $2,5 \mathrm{mg} / \mathrm{kg}$ IV.

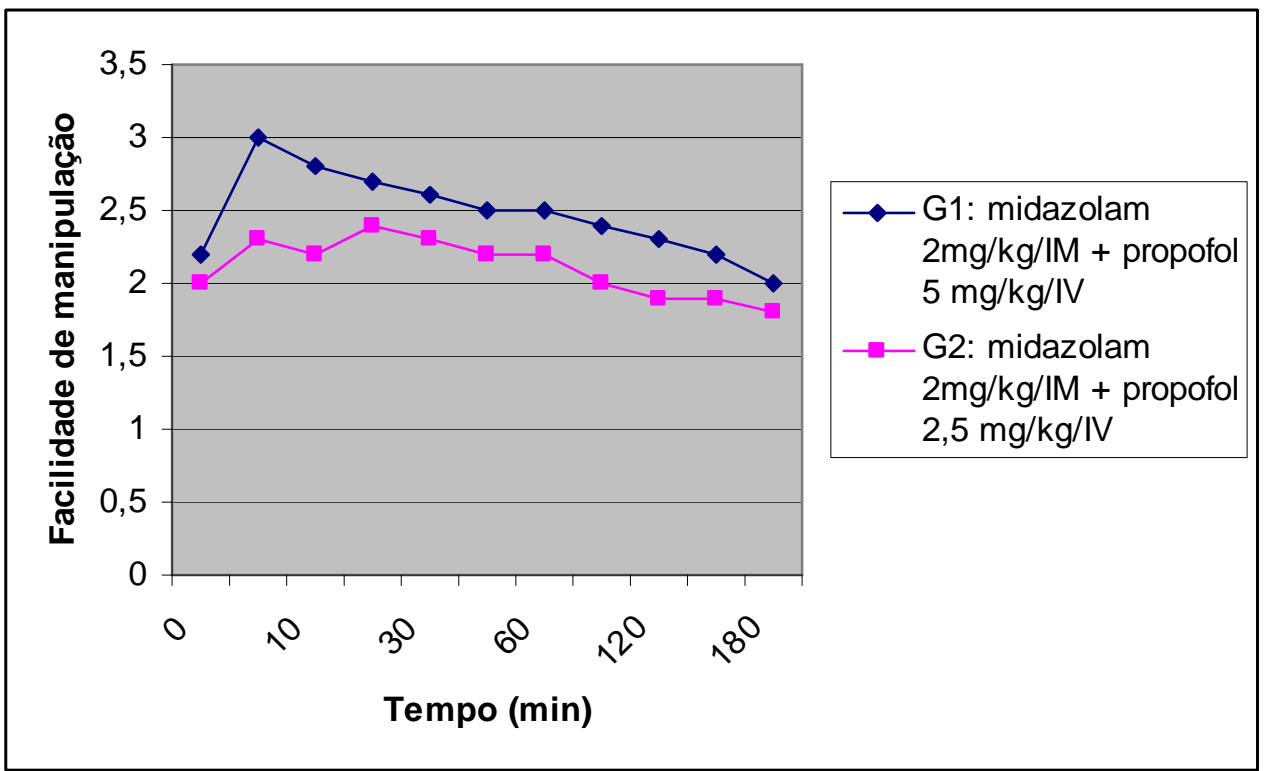

Gráfico 5: Médias dos escores da facilidade de manipualação nos diferentes tempos, em Phynops geoffroamus anestesiadas com as associações midazolam $2 \mathrm{mg} / \mathrm{kg}$ IM e propofol $5 \mathrm{mg} / \mathrm{kg}$ IV e midazolam 2 $\mathrm{mg} / \mathrm{kg} \mathrm{IM}$ e propofol $2,5 \mathrm{mg} / \mathrm{kg}$ IV. 
SANTOS, A.L.Q. et al. Avaliação do uso da combinação de propofol e midazolam na contenção farmacológica e anestesia do cágado-de-barbicha Phrynops geoffroanus SCHWEIGGER, 1812 (Testudines, Chelidae). PUBVET, Londrina, V. 6, N. 12, Ed. 199, Art. 1337, 2012.

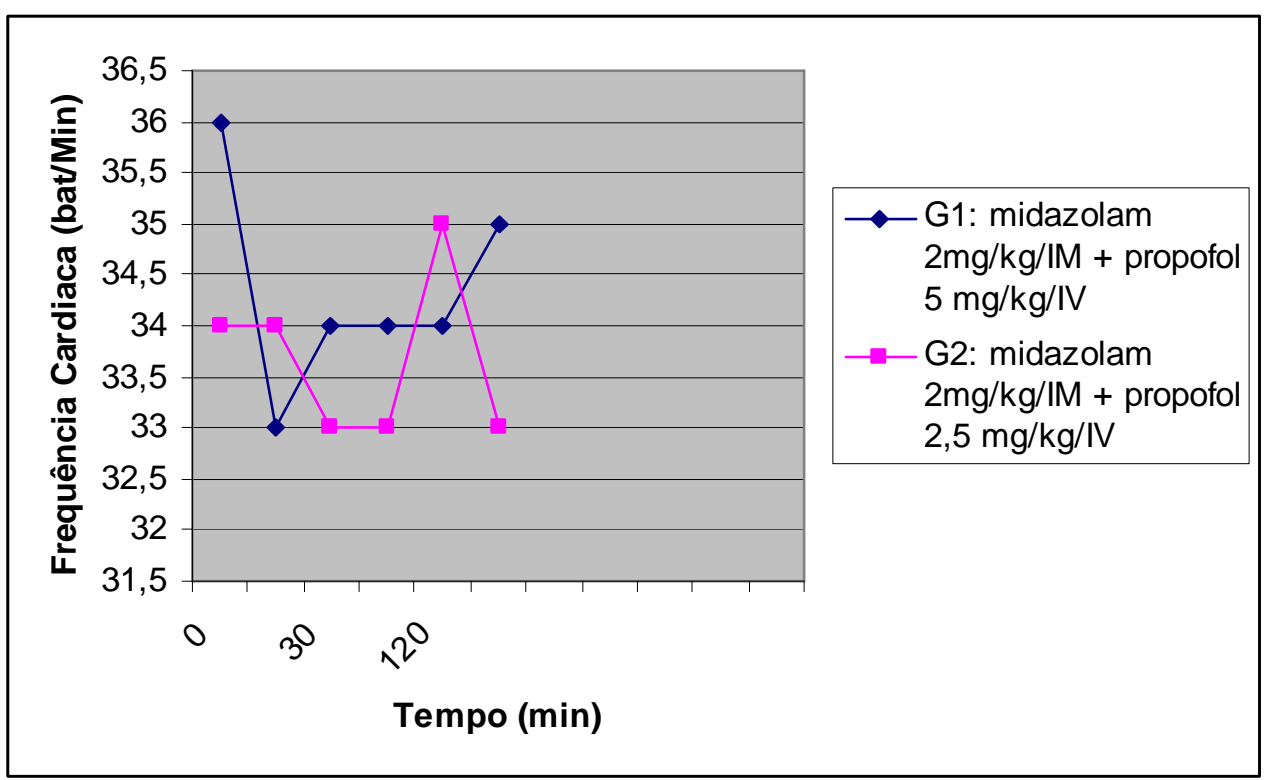

Gráfico 6: Médias dos escores da frequência cardiaca nos diferentes tempos, em Phynops geoffroamus anestesiadas com as associações midazolam $2 \mathrm{mg} / \mathrm{kg}$ IM e propofol $5 \mathrm{mg} / \mathrm{kg}$ IV e midazolam $2 \mathrm{mg} / \mathrm{kg} \mathrm{IM}$ e propofol $2,5 \mathrm{mg} / \mathrm{kg}$ IV.

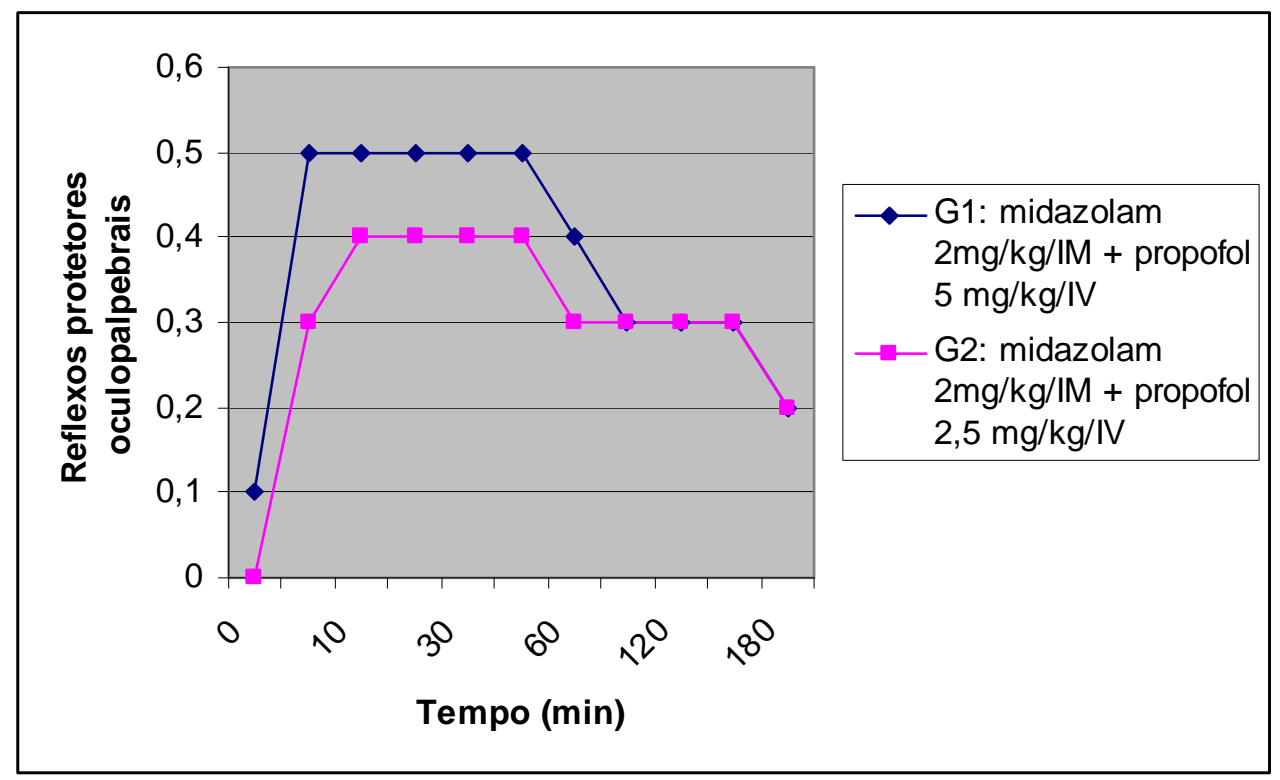

Gráfico 7: Médias dos escores dos reflexos protetores oculopalpebrais nos diferentes tempos, em Phynops geoffroamus anestesiadas com as associações midazolam $2 \mathrm{mg} / \mathrm{kg} I \mathrm{IM}$ e propofol $5 \mathrm{mg} / \mathrm{kg}$ IV e midazolam 2 $\mathrm{mg} / \mathrm{kg}$ IM e propofol $2,5 \mathrm{mg} / \mathrm{kg}$ IV. 
SANTOS, A.L.Q. et al. Avaliação do uso da combinação de propofol e midazolam na contenção farmacológica e anestesia do cágado-de-barbicha Phrynops geoffroanus SCHWEIGGER, 1812 (Testudines, Chelidae). PUBVET, Londrina, V. 6, N. 12, Ed. 199, Art. 1337, 2012.

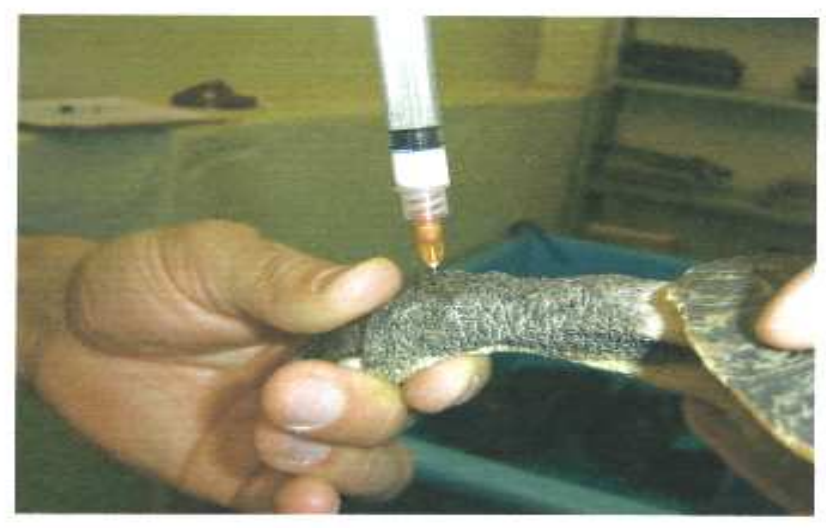

Fotografia I: Aplicação em cágado-de-barbicha (Phrynops geoffroamus), de propofol no seio vertebral cervical.

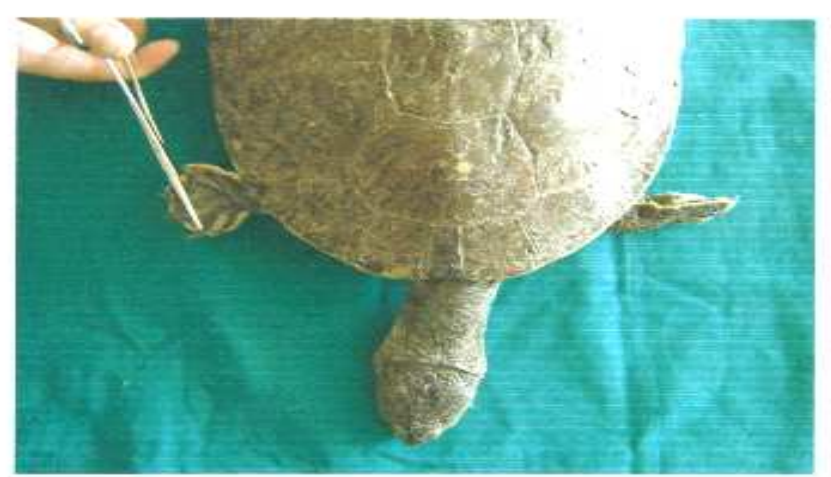

Fotografia 2: Teste para avaliação do estímulo no membro torácico direito, em cágado-de-barbicha (Phrynops geoffroamus), após a aplicação do propofol. 
SANTOS, A.L.Q. et al. Avaliação do uso da combinação de propofol e midazolam na contenção farmacológica e anestesia do cágado-de-barbicha Phrynops geoffroanus SCHWEIGGER, 1812 (Testudines, Chelidae). PUBVET, Londrina, V. 6, N. 12, Ed. 199, Art. 1337, 2012.

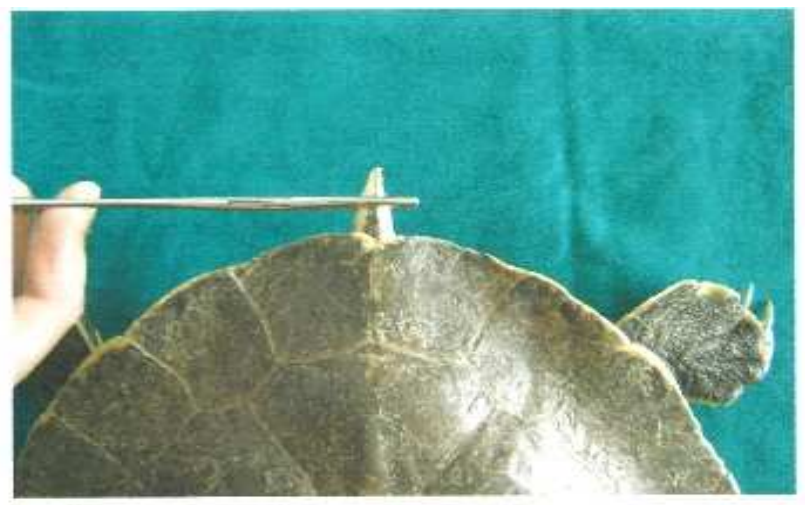

Fotografía 3: Teste para avaliação do estímulo da cauda por pinçamento, em cágado-de-barbicha (Phrynops geoffroanus) após a aplicaçāo do propofol.



Fotografia 4: Cágado-de-barbicha (Phrynops geoffroamus) em grau três de relaxamento muscular, apos a administração de propofol. 
SANTOS, A.L.Q. et al. Avaliação do uso da combinação de propofol e midazolam na contenção farmacológica e anestesia do cágado-de-barbicha Phrynops geoffroanus SCHWEIGGER, 1812 (Testudines, Chelidae). PUBVET, Londrina, V. 6, N. 12, Ed. 199, Art. 1337, 2012.

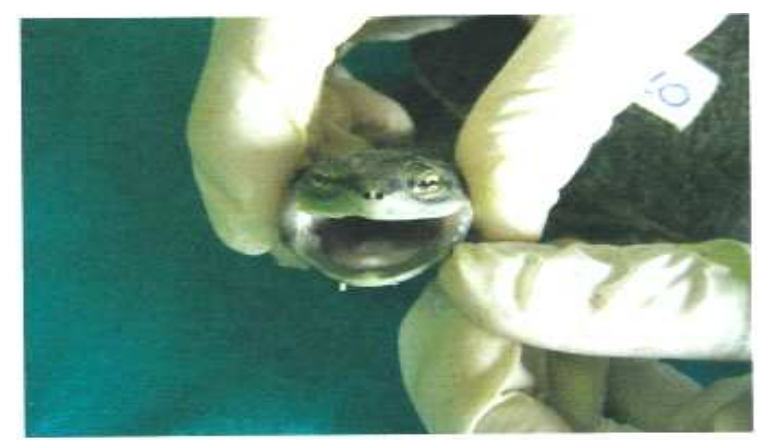

Fotografia 5: Cágado-de-barbicha (Phrynops geoffroanus) em grau três de facilidade de manipulação, após a administração de propofol.

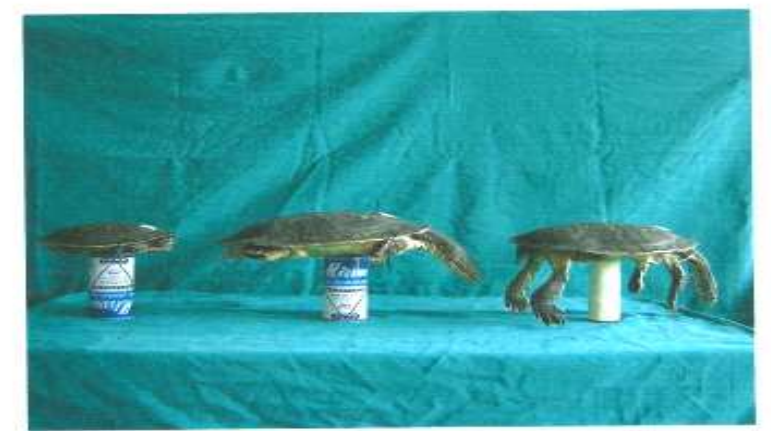
Fotografia 6: Cágados-dc-barbicha (Phrymops geoffroamus)
apresentando, da esquerda para a direita, em grau um, dois e três de sedaçầo, após a aplicação de propofol.

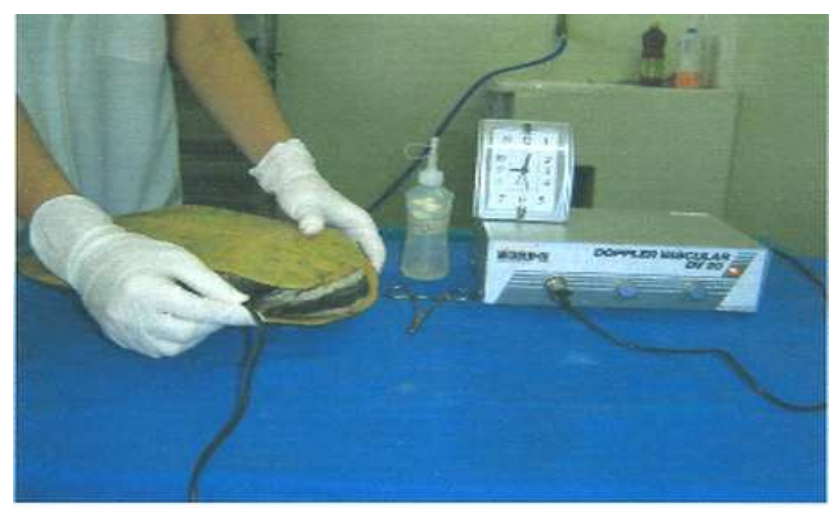

Foto 7: Avaliação da freqüência cardíaca utilizando o Doppler vascular, após a aplicação de propofol em cágado-de-barbicha (Phrynops geoffroamus)

\section{Discussão}

A temperatura ótima para esses répteis apresentarem 0 melhor funcionamento do seu metabolismo oscila entre 28 e $36^{\circ} \mathrm{C}$ (BENNETT, 1991). Os grupos G1 e G2 apresentam temperatura ambiental media de 28 e $31^{\circ} \mathrm{C}$, 
respectivamente. Portanto, foi esperada a melhor ação possível das drogas utilizadas.

No presente experimento, observou-se diferença significativa $(p>0,05)$ nos valores da media de escore locomoção em G2 no tempo 10 minutos, cujos cágados voltaram a se locomover mais rapidamente que os do G1. Resultados semelhantes foram encontrados por Alves Junior (2006) em experimento com Podocnemis expansa, utilizando a combinação de xilazina $1,5 \mathrm{mg} / \mathrm{kg}$ IM e propofol nas doses de 5 e $10 \mathrm{mg} / \mathrm{kg}$ IV, onde observou-se diferença significativa nos tempos 90, 150 e 180 minutos, cujos cágados do grupo com menor dose de propofol $(5 \mathrm{mg} / \mathrm{kg}$ IV) voltaram a se locomover mais rapidamente que os com o maior dose de propofil (10 mg/kg IV) . fato explicado por Magella e Cheibub (1990), que a maior dose de propofol seus efeitos serão prolongados.

Magella e Cheibub (1990) relata que o progresso do relaxamento muscular em repteis anestesiados ocorre no sentido crânio-caudal. Durante a recuperação, a função motora retorna em sentido oposto. O reflexo de endireitamento é perdido mais cedo durante a indução, mais é um parâmetro útil para indicar a recuperação do animal.

A aplicação do midazolam como medicamentos pré-anestésicos produziu adequada sedação nesse experimento, facilitando a aplicação do porpofol no seio vertebral cervical, já que estes quelônios não apresentam reações ao manuseio. Fato explicado por Mosley (2005) que afirma que prémedicamentos facilitam a manipulação e cateterização intravenosa reduzem o estresse do como medicamento pré-anestésico, produziu adequadamente sedação nesse experimento, facilitando a aplicação do propofol no seio vertebral cervical, já que estes quelônios não aparentaram reações ao manuseio. Fato explicado por Mosley (2005) que afirma que pré-medicações facilitam a manipulação e cateterização intravenosa reduzem o estresse da manipulação e os efeitos negativos associados com a administração de altas doses das drogas utilizadas para introdução ou manutenção da anestesia. 
Usando o midazolam, droga que possui como um dos efeitos o relaxamento muscular (MASSONE, 1999), foi observado bom relaxamento muscular no presente experimento, já que tanto os animais de G1 quanto os animais de G2, permanecem durante um período de aproximadamente 120 minutos entre os escores 2 e 3 (Gráfico 2).

Em estudos feitos por Oppenheim e Moon (1995) em Trachemys scripta elegans o midazolam induziu bem o relaxamento muscular reduziu a agressão, e não teve efeitos significativos sobre o sistema cardiorrespiratório. O autor utilizou doses de $1,5 \mathrm{mg} / \mathrm{kg}$ IM a 2,5 mg/kg IM, e contatou neste experimento com dose de $2 \mathrm{mg} / \mathrm{kg}$ IM.

Alves Junior (2006) utilizou a combinação de midazolam e cetamina em Podocnemis expansa. Em seu experimento não foi observado bom relaxamento do muscular nos grupos anestesiados com midazolam (2 mg/Kg IM) e cetamina (20mg/kg IM) e midazolam (2 mg/Kg IM) e cetamina (60 $\mathrm{mg} / \mathrm{kg}$ IM) contrariando os resultados encontrados neste experimento.

Observou-se no presente estudo, que cinco minutos após a aplicação do propofol todos os quelônios do G1 e G2 apresentaram escore Maximo (3) para o relaxamento muscular, permanecendo por aproximadamente 90 minutos com as medidas do escore de relaxamento muscular entre 2 e 3 (Grafico 2). Portanto o relaxamento muscular tanto nos quelônios do G1 quanto nos dos G2 foi satisfatório. Observação que concorda com a afirmação de Duke (1995), onde o relaxamento muscular é uma das principais características desse anestésico injetável o que permite a execução de procedimentos cirúrgicos rápidos.

Pertencente a um grupo de alquilfenóis, o porpol alem de apresentar propriedades hipnóticas em animais, é apropriado para a indução e a manutenção da anestesia (MASSONE 1999). Santana (2004) administrou 10 $\mathrm{mg} / \mathrm{kg}$ IV de propofol em cinco cágados Podocnemis expansa, no seio venoso cervical, e submeteu-os a esofagotomia com sucesso. Pode-se acreditar que esse efeito ocorreu em decorrência de uma acentuada depressão da atividade metabólica cerebral, pois este fármaco induz depressão no sistema nervoso 
central (SNC), por intensificar os efeitos inibitórios de neurotransmissor acido gama-aminobutirico (GAMA), conforme informado por Zanini; Oga (1994).

Foi relatado por Magella e Cheibub (1990) o aumento da analgesia do propofol quando em altas doses, fato não observado neste estudo, já que houveram menos animais apresentando resposta ao estimulo doloroso no membro torácico no grupo utilizando G1 (6 animais) do que no grupo G2 (7 animais). Entretanto, Avila Junior (2005) ao anestesiar Podocnemis expansa , com o propofol associado ao butorfanol e a fentanila, não observou acréscimo a analgesia.

Nesse estudo, ao avaliar a resposta ao estimulo do membro torácico direito, os resultados encontrados em G1 e G2 não foram satisfatórios, já que 6 animais do G1 e 7 animais de G2 não apresentaram resposta ao estimulo. Os quelônios avaliados apresentaram boa facilidade de manipulação, já que eles permanecem por aproximadamente 90 minutos em escores entre 2 e 3 . (Gráfico 5). Em experimento de Alves Junior (2006) ao contrario, não encontrou facilidade de manipulação em seu experimento utilizado midazolam $2 \mathrm{mg} / \mathrm{kg}$ IM e cetamina $20 \mathrm{mg} / \mathrm{Kg}$ IM e midazolam $2 \mathrm{mg} / \mathrm{kg}$ IM e cetamina 60 $\mathrm{mg} / \mathrm{kg} \mathrm{IM}$.

Alves Junior (2006) observou que a dose maior de propofol $(10 \mathrm{mg} / \mathrm{kg}$ IV) utilizada no G2, interferiu diretamente para a redução dos batimentos cardíacos desse cagado. Esse fato é relatado por Magelaa e Cheibub (1990), pois quanto maior sua dose, maior será sua influencia na depressão do aparelho cardiovascular. Goodchild e serão (1993) observaram que o propofol não causou alterações cardiorrespiratórias em cães anestesiados em infusão continua.

Magella e Cheibub (1990) relataram que o propofol é um importante depressor do aparelho cardiovascular. Assim, quando maior for a dose utilizada maior será sua influencia na depressão cardíaca. No entanto, no presente estudo, este efeito não foi observado já que durante o experimento os quelônios não apresentaram diminuição da freqüência cardíaca nas doses de 2,5 e $5 \mathrm{mg} / \mathrm{kg}$. Os mesmos resultados foram encontrados por Alves Junior 
(2006), utilizado xilazina na dose de $1,5 \mathrm{mg} / \mathrm{kg}$ e propofol nas doses de 5 e 10 $\mathrm{mg} / \mathrm{kg}$.

O reflexo corneal (exceto em serpentes) deve estar presente em plano cirúrgico, e quando abolido é sinal de que o paciente esta com o plano anestésico excessivamente profundo (BENNETT, 1996). Neste experimento 5 animais do grupo $\mathrm{G} 1$ que receberam a maior dose de propofol, não perderam os reflexos protetores oculopalpebrais. Já em G2, 7 animais não perderam tais reflexos.

Segundo Trurmon e Tranquilli (1996) a injeção de propofol resulta em alto índice de apneia durante a indução. Resultados similares foram encontrados por Avila Junior (2005), onde todas as Podocnemis expansa estudadas apresentaram apneia, tendo como sintomas a cianose da mucosa oral e língua. A pesar dos quelônios de seu experimento manifestarem apneia de longa duração e não receberam ventilação assistida, nenhum veio a óbito, pois conforme Bennett (1991) os repteis são capazes de permanecer por tempo prolongado em apneia podendo fazer respiração anaeróbica. No entanto no presente estudo não observou - apneia nos cagados pois para evitar este problema, utilizou-se tempo de aplicação longo do propofol, aproximadamente 1 minuto.

Em situações nas quais a administração de propofol é precedida de medicação pré-anestésica, a dose de indução pode ser reduzida entre 25 e 75\% (GEEL, 1991; HALL et AL. 1999). Protocolo utilizado midazolam como MPA e propofol foi utilizado neste estudo em $P$. geoffronus, obtendo-se sedação satisfatória, porem sem diferença significativa entre os grupos experimentais.

\section{CONCLUSÃO}

A associação anestésica de midazolam $2 \mathrm{mg} / \mathrm{kg}$ IM e propofol $5 \mathrm{mg} / \mathrm{Kg}$ IV e midazolam $2 \mathrm{mg} / \mathrm{Kg}$ IM e propofol $2,5 \mathrm{mg} / \mathrm{Kg}$ IV não são indicados para procedimentos cirúrgicos em Phrynops geoffroanus. 
SANTOS, A.L.Q. et al. Avaliação do uso da combinação de propofol e midazolam na contenção farmacológica e anestesia do cágado-de-barbicha Phrynops geoffroanus SCHWEIGGER, 1812 (Testudines, Chelidae). PUBVET, Londrina, V. 6, N. 12, Ed. 199, Art. 1337, 2012.

Ambas as associações foram eficazes para sedação nestes animais, o que possibilita a contenção farmacológica, coleta de amostras e exames físicos.

Ambas as associações foram eficazes para promover um bom relaxamento muscular e facilidade de manipulação, portanto é possível utilizar a menor dose de propofol $(2,5 \mathrm{mg} / \mathrm{kg})$ e obter efeitos satisfatórios.

\section{REFERÊNCIAS}

ALVES JÚNIOR, J.R.F. Anestesia de tartarugas-da-amazônia (Podocnemis expansa) com as associações de cetamina e propofol, midazolam e propofol e midazolam e cetamina. 2006. 43 f. Dissertação (Mestrado) - Faculdade de Medicina Veterinária, Universidade Federal de Uberlândia, Uberlândia, 2006.

AVILA JUNIOR, R.H. Efeitos anestésicos do propofol e do etomidato isolados e associados com butarfonol e fentanila em tartaruga-da-amazônia (podocnemis expansa-Schweigger,1812). 2005. 31f Dissertação (Mestrado) - faculdade de Medicina Veterinária, Universidade Federal de Uberlândia, Uberlândia, 2006.

BENNETT, R.A A review of anesthesia and chemical restrain in reptiles. Journal of Zoo and Widlife Medicine, Philadelphia, v. 22, p. 282-303, 1991.

DUKE, T. A new intravenous anesthetic agent: Propofol. Canadian Veterinary Journal, Ottawa, v.36, p.181- 183, 1995.

ERNEST, C.H.; BARBOUR, R.W. Turtles of the world. Washigton: Smithsonian Institution, 1989. $383 \mathrm{p}$.

GARCIA-NAVARRO, C.E.K; PACHALY, J.R. 1994. Manual de hematologia veterinária, São Paulo: Livraria Varela, 1994. 169.

GELL, J.K. The effect pf premedication on the induction dose of propofol in dogs and cats. Journal of the South African Veterinary Association, Pretoria, v.62, n. 3, p. 118-123, 1991.

GOODCHILD, C.S.; SERRÃO, J.M. Cardiovascular effects of propofol in the anaesthetized dog. British Journal of Anaesthesia, Oxford, v. 63, p. 87-92, 1993.

HAAL, T. L.; DUKE, T.; TOWSRND, H.G.; CAULKETT, N.A.; CANTWELL, S.L. The effect of opioid and acepromazine premedication on the anesthetic induction dose of propofol in cats.

Canadian Veterinary Journal, Ottawa, 1999, v.40, n. 12, p. 867-870.

INSTITUTO BRASILEIRO DO MEIO AMBIENTE E DOS RECURSOS NATURAIS RENOVAVEIS. Projeto quelônios. Brasília, 2004. 119 p.

MAGELLA, H.A.; CHEIBUB, Z.B PROPOFOL: Propofol: revisão bibliográfica. Revista Brasileira de Anestesiologia, Rio de Janeiro, v. 40, n. 4, p. 289-294, 1990. 
MASSONE, F. Medicação pré-anestésica. In:

Anestesiologia veterinária:

farmacológica e técnicas. 3. Ed. Rio de Janeiro: Guanabara Koogan, 1999. P. 17-30.

MOSLEY, C.A.E. Anesthesia and analgesia in reptiles. Seminars in avian and exotic pet medicine, v. 14, n. 4, p. 243-262, Oct/ 2005.

OPPENHEIM, Y.C.; MOON, P.F. Sedative efects of midazolam in red eared slider turtles (Trachemis scripta elegans). Journal of Zoo Wildlife Medicine, Philadelphia, v. 26, n. 3, p. 409-413, 1995.

ORR, R. T. Biologia dos vertebrados. 5.ed. São Paulo: Rocca, 1986. 508 p.

POUGH, F.H; HEISER, J.B.; MCFARLAND, W.N. A vida dos vertebrados. 2.ed. São Paulo: Atheneu, 1999. 356 p.

SANTANA, E. P. S. Esofagotomia cervical em tartaruga-da-amazônia (Podocnemis expansa - Schweigger, 1812) (Testudines - Pelomedusidae). 2004. $54 \mathrm{f}$. Trabalho de conclusão de curso (Graduação). Faculdade de Medicina Veterinária. Universidade Federal de Uberlândia: 2004.

SOUZA, F.L. Uma revisão sobre padrões de atividade, reprodução e alimentação de cágados brasileiros (testudines, Chelidae). Phyllomedusa: Revista de Herpetologia Neotropical, Belo Horizonte, v. 1, n. 3. p. 15-27. 2004.

THURMON, J.C.; TRANQUILLI, W.J. Injectable Anesthetics In: Lumb \& Jones

Veterinary Anesthesia. Pennsylvania: Saunders, 1996. P 210-240.

VILLE, C.A.; WALKER JÚNIOR, N.F.; BARNES, R.D. Zoologia geral. 6ed. Rio de Janeiro Interamericana, $1985.683 \mathrm{p}$.

ZANINI, A.C.; OGA, S. Farmacologia aplicada. 5.ed. São Paulo: Atheneu, 1994. 739 p. 